\title{
EL “NUEVO PENSAMIENTO” DE FRANZ ROSENZWEIG: UNA FILOSOFÍA DEL DIÁLOGO Y DE LA EXPERIENCIA
}

\author{
José Luiz Bueno \\ Licenciado en Filosofía por la Universidad de São Paulo (USP) - Brasil, \\ Magíster en Filosofía por la Pontificia Universidad Católica de Valparaíso \\ Email: jluizb@yahoo.com.br
}

\section{RESUMEN}

Franz Rosenzweig (1886-1929) fue un importante pensador alemán de los comienzos del siglo XX, cuya principal obra es La Estrella de la Redención. Rosenzweig se enfrentó al colapso del idealismo y en su obra desveló el estado de fragmentación del pensamiento filosófico en su tiempo. Sobrepasando su propio hegelianismo y los sistemas idealistas de la totalidad, dedicó su pensamiento de la madurez al desarrollo de un sistema de filosofía que valoró la singularidad humana en toda su plenitud existencial.

Esta singularidad es un elemento inasible por el sistema idealista de la totalidad pues que se queda fuera de este sistema. Precisamente por ello es que Rosenzweig le atribuye importancia filosófica dado este su aspecto existencial.

Muchos investigadores contemporáneos de su obra lo sitúan entre los existencialistas, sin embargo Rosenzweig va más allá del existencialismo pues él percibe el Hombre en su relación dialógica con los otros elementos que constituyen la realidad, que son Dios y el Mundo. El hecho de la existencia es revelado en las relaciones y sólo puede ser pensado por un Hombre concreto, jamás por un Hombre ideal. Así, consideramos en este artículo dos de los rasgos más relevantes la filosofía de Rosenzweig, que es un pensamiento de la experiencia y de la existencia, que se constituye en la relación con el otro a través del diálogo y del habla, en el tiempo.

Palabras-clave: Franz Rosenzweig, existencia, diálogo, perdón, experiencia, idealismo.

\section{ABSTRACT}

Franz Rosenzweig (1886-1929) was an important German thinker of the early twentieth century, whose main work is The Star of Redemption. Rosenzweig faced the collapse of idealism and his work revealed the fragmented state of philosophical thought in his time. Surpassing his own Hegelianism and the idealistic systems, he dedicated his late thinking to develop a system of philosophy that valued human uniqueness in all its existential fullness. 
This singularity is an element that is unattainable by the idealistic system of totality because it falls outside of this system. Precisely for this reason is that Rosenzweig attaches philosophical importance due to its existential aspect.

Many contemporary scholars place his work among the existentialists, however Rosenzweig goes beyond existentialism as he sees Man in his dialogical relationship with the other components of the reality, which are God and the World. The fact of existence is revealed in their relationships and can only be thought of by a particular man, and never by an ideal man. Thus, we consider in this article two of the most important features of Rosenzweig's philosophy, which is a thought of the experience and existence, which is in relation to the other through dialogue and speech over time.

Keywords: Franz Rosenzweig, existence, dialogue, forgiveness, experience, idealism.

\section{El "Nuevo Pensamiento" de Franz Rosenzweig: una filosofía del diálogo}

El filósofo Franz Rosenzweig nació el año 1886 y falleció tempranamente en 1929. Tras obtener su grado doctoral en 1912 con la tesis Hegel y el estado ${ }^{1}$, Rosenzweig vivió el horror y el impacto de la Primera Guerra Mundial como soldado en la frente alemana de los Balcanes. Desde allí, escribiendo en tarjetas postales enviadas a su casa en Alemania y copiadas por su madre, Rosenzweig compone su opus magna, llamada La Estrella de la Redención, publicada en 1921. Además de esa obra, aún durante la guerra Rosenzweig escribió una gran cantidad de cartas en las cuales consignó importantes discusiones teóricas que constituyen parte fundamental de su pensar. Un ejemplo es la correspondencia con su amigo Eugen Rosenstock-Huessy, en la cual los dos interlocutores desarrollan un verdadero diálogo entre el judaísmo y el cristianismo ${ }^{2}$.

Con el fin de la guerra, Rosenzweig pudo dedicar su tiempo a los temas que realmente le cautivaran todo su interés. Con base en un significativo cambio en sus inquietudes filosóficas y religiosas y en conclusiones a las que había llegado, Rosenzweig declina la oferta de una posición de profesor en la Universidad de Berlín. En una carta a su mentor, Rosenzweig explica que ni la curiosidad científica ni el apetito estético ya no le dicen nada ${ }^{3}$. Con este giro existencial, Rosenzweig dice que ahora le interesa preguntar cuando le preguntaren, o sea, cuando quien le dirigir preguntas sean hombres y no eruditos, señalando así la importancia en su pensamiento y en su vida del diálogo entre hombres vivos, singulares, existentes. Es este ethos de servicio al hombre que lo llevará a producir una obra filosófica de gran originalidad.

\footnotetext{
1 ROSENZWEIG, Franz. Hegel und der Staat. Druck und Verlag von R. Oldenburg, München und Berlin, 1920

2 Esta correspondencia fue publicada por Eugen Rosenstock-Huessy en ROSENZWEIG, Franz, ROSENSTOCK-HUESSY, Eugen. Judaism despite Christianity. The "Letters on Christianity and Judaism" between Eugen Rosenstock-Huessy and Franz Rosenzweig. ROSENSTOCK-HUESSY, Eugen (ed.). University of Alabama Press, Alabama, 1969.
} 
Tras la publicación de La Estrella de la Redención, se hizo necesario escribir a un texto a modo de prefacio tardío, El Nuevo Pensamiento ${ }^{4}$, en el cual presenta los rasgos más importantes de su gran obra, y cuyo título pasa a designar su pensamiento como un todo. Este texto contiene gran parte de las ideas aquí expuestas.

En sus obras siguientes a la tesis, Rosenzweig pasa a aclarar su ruptura con Hegel y con toda la tradición del idealismo. En el pensamiento que pasa a desarrollar, Rosenzweig critica la concepción idealista de una totalidad debido que ella presenta una completa incapacidad de hacerse cargo de la singularidad, del existente humano. Esta critica Rosenzweig ya la había encontrado en pensadores como Schelling, Schopenhauer, Kierkegaard o Nietzsche. Nuestro filósofo la identifica y a ella agrega su propia contribución a este "nuevo pensamiento", que busca comprender la singularidad, el existente humano, y entender su peso ontológico y su consistencia filosófica.

Rosenzweig propone que el pensamiento no se funda a si mismo sino que es fundado "metalogicamente" en otro - el meta-lógico en Rosenzweig significa algo que se fundamenta no en la lógica producto del pensamiento racional sino que en algo que es anterior al pensamiento, anterior a la razón y a la lógica-, más específicamente en el complejo de la realidad existencial. El verdadero pensamiento no permanece en si mismo, sino que señala a la realidad existencial a la cual pertenece. El pensamiento de Rosenzweig dirige su atención hacia el individuo, el ser humano que sufre, que ama, que se equivoca, que espera y desespera, el mismo ser humano tan descuidado por el sistema clásico de pensamiento que lo hace desvanecerse en el Todo.

Juntamente con dar plena importancia al individuo, al singular, el nuevo pensamiento de Rosenzweig otorga preeminencia al tiempo, al instante, a la vida, y trata de recuperar la primacía ontológica de la pluralidad, de la contingencia, de la particularidad.

El pensamiento respecto el individuo gana importancia especial para Rosenzweig a partir de su experiencia de la guerra por medio de la cual la muerte se le hizo extremadamente patente. Rosenzweig se dio cuenta del esfuerzo llevado a cabo por el pensamiento idealista para librar el hombre del temor a la muerte. Este esfuerzo pasa exactamente por el intento de demostrar la pertenencia del hombre a una totalidad que lo constituye y lo fundamenta. Según el concepto idealista, una totalidad no muere, por ende el hombre perteneciente a ello y fundamentado en el Todo tampoco muere. Como dice Rosenzweig, según la lógica idealista "un todo no ha de morir, y en un todo nada moriría. Sólo lo aislado puede morir" ${ }^{5}$.

Pero este hombre concebido por el idealismo no es el mismo que el hombre que muere. $Y$ el hombre que muere no es abarcado ni comprendido por el sistema idealista. No obstante el esfuerzo de toda la tradición idealista, la muerte sigue imponiéndose en toda su materialidad y como signo máximo de la individualidad y singularidad del ser humano existente, vivo. Sin embargo, a pesar de todo el esfuerzo idealista de hacer de la muerte una nada, ocultándola en la niebla del concepto del Uno-Todo, a pesar de pretender haberla hundido en la noche de la nada,

4 ROSENZWEIG, Franz. El nuevo pensamiento. Visor, Madrid, 1989. Trad. Isidoro Reguera y Francisco Jarauta

5 Rosenzweig, Franz. La Estrella de la Redención. Trad. de Miguel García-Baró. Salamanca: Sígueme, 2006. p. 44 
dice Rosenzweig que la angustia del hombre ante la muerte sigue desmintiendo lo que él llama "la mentira piadosa y compasiva de la filosofía"6.

Esta consciencia de que hay el individuo, el ser singular en su existencia, el particular que se pone delante y fuera del sistema de la totalidad, consciencia puesta de manifiesto por el hecho irreductible e irrefutable de la muerte, es el primer y también el gran paso de Rosenzweig para hacer notoria la fractura del sistema de la totalidad. El hombre, que se cuestiona a sí mismo, se pone fuera del sistema de la totalidad y se reconoce a sí mismo como extrasistemático, o, para usar el prefijo preferido por Rosenzweig, meta-sistemático.

Precisamente cuando el Hombre se pone fuera del Todo, lo mismo pasa con los otros dos elementos primordiales, o Elementales, de la realidad que son el Mundo y Dios. Los tres aparecen, ahora, en su singularidad, tal como son dados en la experiencia existencial, en su positividad concreta.

Rosenzweig asume, así, una posición claramente opuesta a la tradición filosófica que, en sus propios términos, va desde Tales hasta Hegel, desde Jonia a Jena, o sea, al idealismo. La oposición fundamental de Rosenzweig a esta tradición se refiere al hecho que el idealismo, en su intento de abarcar la realidad bajo un visión de totalidad, no logra comprender ni abarcar la vida individual, la singularidad humana, lo particular, lo viviente. Rosenzweig se opone al concepto idealista de un Todo-Uno lo cual tendría un carácter inteligible. La pregunta de la filosofía tradicional por la esencia de las cosas la hace alejarse de la cosa misma, la impide de ver la cosa real. Eso, Rosenzweig lo considera la enfermedad del filósofo. Cuando el filósofo pregunta por las esencias,

"El mundo no puede ser en modo alguno el mundo, Dios en modo alguno Dios, el hombre en modo alguno hombre, sino que 'propiamente' todos han de ser algo completamente diferente. ¡Si no fueran algo diferente, sino sólo lo que realmente son, la filosofía (¡Dios nos libre!) resultaría al final superflua! Al menos una filosofía que se empeñara a todo trance en descubrir algo completamente diferente" ${ }^{\text {. }}$.

Rosenzweig denuncia el otro movimiento de la filosofía tradicional que es la reducción de los tres elementos fundamentales a uno de ellos. Este movimiento se presenta bajo la forma de momentos históricos del pensamiento filosófico: la filosofía antigua redujo Dios y el Hombre al Mundo, la cual es la perspectiva cosmológica; la Edad Media redujo el Hombre y el Mundo a Dios, en la perspectiva teológica; la Edad Moderna ha reducido Dios y el Mundo al Hombre, en la perspectiva antropológica.

Pero, tras la fractura del todo, queda evidente que estos tres elementos no pueden ser reducidos uno al otro. Rosenzweig, en una perspectiva positiva, dice que, en verdad, los tres solo pueden ser reducidos a sí mismos. Así que, en el Mundo, solo se va encontrar mundanidad; en Dios, solo divinidad; en el hombre, solo humanidad. Y solo en el mundo se va encontrar mundanidad, solo en el hombre la humanidad y solo en Dios la divinidad. Rosenzweig trata así de descubrir aquello que no necesita ser pensado para ser, el ser que está más allá del pensar, indicado por el prefijo 
"meta". Así, estos tres elementos fundamentales remiten a los objetos irracionales, o extrarracionales, que los sustentan y les dan sentido: el Hombre metaético, el Mundo metalógico y el Dios metafísico.

Se nota ahí el despegue operado por Rosenzweig entre el ser y el pensar. Para nuestro autor, el error de la filosofía tradicional no está en la respuesta dada a la pregunta "qué es", sino que el error está en la pregunta misma, cuya respuesta será una esencia, una realidad distinta de cada uno de estos elementos, saliendo fuera de ellos mismos, llevando a algo que no son ellos mismos. Rosenzweig compara estos tres elementos a cebollas ${ }^{8}:$ por mucho que se las pele, tras cada capa solamente se llegará a otra capa de cebolla, nunca a algo diferente de ella.

Estos tres elementos tampoco pueden ser demostrados por medio de la razón sino tan solo revelados a través de la experiencia. Sus naturalezas propias los sitúan no un al lado del otro sino que un frente al otro, o sea, estos elementos se revelan no por lo que son en si mismos mas por las relaciones que establecen unos con los otros.

A la existencia concreta, así como todo lo que es original, irrepetible, no se accede por deducción; la existencia concreta no puede se aprehendida de otro modo sino que solamente por la experiencia. La experiencia debe ser, por lo tanto, el punto de partida del nuevo pensamiento.

El nuevo pensamiento de la experiencia de Rosenzweig solamente puede ser comprendido en su conexión con su crítica al idealismo filosófico y a su unidimensionalidad y estrechez en cuanto una teoría de la consciencia. En La Estrella de la Redención, Rosenzweig ofrece una importante alternativa metodológica al método especulativo del idealismo hegeliano.

Su método no empieza desde el Ser como el pensamiento mas simple y abstracto respecto la totalidad absoluta y única. Su método, en cambio, empieza con un triple no-saber frente a los tres elementos primordiales, las tres "factualidades" de la filosofía de la experiencia: Dios, el mundo, el Hombre.

En vez de un despliegue de una totalidad que anticipa su conclusión ya en el principio, asimismo que en cada movimiento particular de su despliegue, Rosenzweig presenta un movimiento no uniforme de los tres elementos primordiales de la realidad. Aquí no hay homogeneidad, tampoco el movimiento circular del pensamiento especulativo. Rosenzweig critica la linealidad de las sucesivas afirmaciones y negaciones del movimiento metodológico idealista. Con Rosenzweig, el pensamiento adquiere una multiplicidad además de una novedad a cada movimiento. En vez de una forma auto-cerrada de pensamiento, un método de experiencia. ${ }^{9}$

La preeminencia que Rosenzweig atribuye a la experiencia no quiere decir que él esté intentando reducir la ciencia a un mero empirismo ni tampoco que quiere abandonar la forma científica de la filosofía. Lo que sí él quiere es delimitar la reivindicación de universalidad del pensamiento científico tomando en consideración el hecho de la experiencia ${ }^{10}$.

8 Rosenzweig, El Nuevo Pensamiento, p. 50

9 WIEHL, Reiner. Experience in Rosenzweig's New Thinking, in, The Philosophy of Franz Rosenzweig, pp 51-52

10 Experience in Rosenzweig's New Thinking, pg 55

Cuadernos Judaicos-ISSN: 0718-8749 
Estos cambios en la relación de la experiencia con la ciencia hacen la experiencia alcanzar independencia. Además de la experiencia científica, hay una experiencia no-científica, que no se reduce a una forma pre-científica de conocimiento. Ella es algo diferente. Cada experiencia porta en si su constitución divina, mundana o humana. Dios, el Mundo, el Hombre, son para Rosenzweig hechos primordiales de la experiencia, factualidades, dados en la experiencia antes de toda objetividad y de toda consciencia de ellos. Por esta misma razón, por ser pre-objetivos, ellos penetran la experiencia con mucho más fuerza que cualquier objetividad y consciencia imaginable ${ }^{11}$.

El empirismo de esta filosofía tiene un carácter y un significado muy distinto de lo usual. El empirismo aquí se refiere a aprehender la existencia en su aspecto concreto. De esta manera, el sujeto da la experiencia puede ser tan solo el hombre en la plenitud de su ser y no solamente en su órgano epistemológico. El hombre, individual y efectivo, es sujeto y objeto de la experiencia.

No se tiene experiencia "de" la facticidad sino que "en" la facticidad, en las relaciones que se establecen entre los elementales. Los elementales sólo se abren en sus relaciones, sólo en la creación, en la revelación y en la redención. ${ }^{12}$

El conocimiento de los elementos es proporcionado por la experiencia, por la vivencia, por el "saber intuitivo", que es un saber previo que hace posible hablar de los elementos. En cierto sentido, Rosenzweig aúna, de esta manera, la creencia con el conocimiento. No se conoce el ser de los elementos, pero sí su dimensión positiva. Como dice Rosenzweig, "lo que hacen o lo que se hace con ellos",13.

La experiencia es la única vía de acceso al real. Esto se indica en la obra La Estrella de la Redención en la presentación de Rosenzweig de la realidad en forma narrativa, tal como aparece en la experiencia.

Este concepto de narrativa Rosenzweig lo obtuvo en el pensamiento del último Schelling, en el fragmento Las Edades del Mundo ${ }^{14}$, ya en la Introducción de la obra. Cabe destacar que Schelling es un influjo fundamental en la composición de La Estrella de la Redención. En una carta a su primo Hans Ehrenberg, Franz Rosenzweig dice que si Schelling hubiese completado la obra Las Edades del Mundo, su libro La Estrella de la Redención no tendría ningún mérito ${ }^{15}$. Tómese como ejemplo la estructura misma de la obra, la cual, inspirada en la propuesta del fragmento de Schelling, se divide en tres tomos: Creación, Revelación, Redención, que siguen la orden del tiempo en pasado, presente y futuro. En el último Schelling, Rosenzweig encuentra los elementos de la crítica al pensamiento de la totalidad y las intuiciones fundamentales respecto a la necesidad de un sistema de filosofía que sea consciente de lo existente, de lo vivo, de lo particular, de lo irracional - en el sentido de ser un más allá de la razón. Los dos primeros libros de La Estrella de

\footnotetext{
11 Idem, pg 58

12 El nuevo pensamiento, p. 61

13 El Nuevo Pensamiento, p. 60, 72

14 SCHELLING, F.W.J, Las Edades del Mundo. Cerro Alegre, Santiago, 1993. Trad. Hugo Renato Ochoa.

15 Apud Franz Rosenzweig Philosophical an Theological Writings, ed., coment. and notes by Paul W. Franks and Michael L. Morgan. Hackett Publishing Company, Inc., Indianápolis, 2000, p. 38 
la Redención fueran desarrollados en acuerdo a las categorías de filosofía negativa y filosofía positiva propuestas por Schelling.

A través del concepto de narrativa, Rosenzweig hace sobresaliente la importancia de la "palabratiempo", o sea, el verbo. Su método puede así mostrar una de sus diferencias fundamentales pues ya no se trata de "pensar" sino que de "hablar". Con el habla, el tiempo es insertado en el proceso. El pensamiento abandona la intemporalidad del método de la filosofía tradicional, que requiere que uno piense solo. Rosenzweig hace notar que el pensamiento no establece diálogo. El pensador ya sabe lo que quiere decir y solo trata de encontrar una manera de decirlo. El pensamiento queda cerrado en sí mismo, no se dirige a nadie. De manera distinta de los diálogos escritos, como por ejemplo los diálogos platónicos, donde ya se sabe previamente las objeciones que serán presentadas, el método del nuevo pensamiento se nutre del tiempo e del otro. En el diálogo basado en el habla, uno no sabe lo que el otro le va a contestar, en verdad, uno no sabe ni siquiera lo que va a decir a continuación. El que habla necesita que el otro lo solicite. Este otro puede representarse de distintas maneras; el otro es el "oyente" en la narración (el pasado), el otro es el "interlocutor" en el diálogo (en el presente); el otro, en el futuro, es el "contertulio"16 (en el coro). El dialogo es la modalidad central del lenguaje y su importancia se hace patente en el sentido de que a través de ello se explicita la constitución del yo. En el dialogo aparece la heteronomía del yo. El yo no se constituye desde sí mismo sino desde el "tú", es decir, desde el otro, que es la heteronomía. El yo, para se constituir, depende de un tú que le es anterior.

Esta prioridad del tú sobre el yo Rosenzweig la presenta en la pregunta bíblica, dirigida por Dios a Adam, al hombre: “¿dónde estás tú?" Desde su escondite, el hombre contesta: “¿soy yo responsable?". En este relato, leído por Rosenzweig según su método narrativo, él quiere demostrar primero que el hombre se hace un yo cuando es despierto a la existencia tras el diálogo con el tú divino - la Revelación - y, segundo, que el individuo existente se constituye sujeto moral no cuando sométese y protesta su inocencia sino cuando admite su responsabilidad. Entonces, se comprende que la subjetividad no se funda en la autonomía sino en la responsabilidad que viene de fuera. O sea, nos descubrimos cuando nos sentimos vistos por el otro: "como has sabido que estabas desnudo?", es decir, nos reconocimos culpables porque estamos en presencia del otro. Este sentido del dialogo tiene que ver con la articulación de la relación Yo-Tú que Rosenzweig desarrolló juntamente con Martin Buber.

La Revelación es el instante en el cual la subjetividad, el Sí-mismo se hace alma. El alma es el individuo despierto para la consciencia de la existencia. Pero el despertamiento no es un acto auto producido sino que es el resultado del amor del tú divino por el yo humano. La presencia del tú divino es el agente que causa que el alma despierte para el presente de su existencia singular. En el capitulo Revelación de la Estrella de la Redención, Rosenzweig propone que el alma se sabe culpable y se avergüenza de este tiempo en que no se sabía una singularidad, se avergüenza de "no haber roto con sus propias fuerzas la proscripción en que estaba"17. Este período en que no conocía el amor del tú divino y en que no se sabía un alma le provoca a ella la consciencia de su culpabilidad, de su pecado ${ }^{18}$. La experiencia del amor de Dios aún no es suficiente para que el

\footnotetext{
16 El Nuevo pensamiento, p 62. Contertulio = compañero de conversación. Tertulia = grupo de personas que se reúne habitualmente para conversar o entretenerse.

17 Estrella, pg. 225

18 lbíd.
}

Cuadernos Judaicos-ISSN: 0718-8749

№28 diciembre 2011 
alma salga completamente de la clausura de su mismidad pues el amor divino tiene que desbordarse hacia el otro, el tú humano.

El lenguaje expresa estos momentos a través de las formas del diálogo yo-tú que empieza en la Revelación. Y la Revelación es el paso necesario para que Hombre y Mundo caminen hacia el encuentro con el Uno, Dios. La responsabilidad que ahora se hizo clara para el sí-mismo despierto, el alma, es que ella debe hacerse cargo de la venida del Reino, que es la Redención final. Un alma despierta es libre para amar a Dios y al próximo, y por tanto, por desear y pedir y esperar la venida del Reino. La Redención final, el perdón del pecado de la inconsciencia existencial y del alejamiento de Dios sólo será perdonado a través de la reunión de Hombre y Mundo por la Redención, que es el momento en que se encuentran la Humanidad y Dios. Dice Rosenzweig que el Reino de Dios "no es sino, precisamente, la mutua unión del alma y el mundo entero" ${ }^{19}$.

La Redención final, el perdón divino, depende que el Hombre primero sepa que hay el "otro" humano y después que estos "yo" despiertos se aúnen por el amor en la plegaria por la venida del Reino. La forma del lenguaje de lo yos unidos es el coro. El coro son las voces que antes dialogaron y que ahora se hacen una voz que se alza a Dios clamando por la venida del Reino, 0 sea, por el encuentro de Hombre y Mundo con la eternidad.

La Redención, el perdón universal, es el producto de acto libre y obediente de los hombres libres que, despiertos por la Revelación, se aunaran por el amor, redimiendo de esa manera el Mundo.

Las formas del lenguaje están sumergidas en el tiempo. El diálogo, que es la forma de la Revelación, está sumergido en el presente, en el instante. El coro, que son las voces aunadas, es la forma del lenguaje de la esperanza, por tanto, el lenguaje que mira hacia el futuro.

La necesidad del tiempo significa la imposibilidad de anticipar nada en la conversación. El pensamiento antiguo se caracteriza por la lógica, el nuevo pensamiento por la gramática. Pensar es pensar para nadie; hablar es hablar a alguien ${ }^{20}$. Obviamente que el pensar hablante sigue siendo un pensar, mas que requiere la presencia de un otro, que no solo escucha sino que también habla. Así, el diálogo se presenta como el método del nuevo pensamiento, pues que es temporal por naturaleza.

A través del método del diálogo, o sea, del habla en el tiempo, el filósofo puede superar el asombro que le es propio y libertarse de la parálisis del pensamiento estático. El que piensa de eso modo sabe que es temporal. Y el tiempo requiere la paciencia para la comprensión. Incluso en la lectura del texto filosófico, Rosenzweig considera que es necesario la paciencia a la vez que recomienda que el lector no se quede paralizado ante las dificultades que el texto presenta, sino que el lector siga leyendo, siga avanzando hasta el final pues solo así la comprensión empieza a aparecer. No hay que detenerse ante las dificultades, ante la falta de claridad; hay que seguir avanzando confiando en el suelo que soporta la experiencia, confiando en el "quizá",1 que representa la multiplicidad de posibilidades que se pueden presentar en el camino abierto adelante.

\author{
19 lbíd., p. 283 \\ 20 El Nuevo pensamiento, p. 63 \\ 21 Estrella, p. 126 \\ Cuadernos Judaicos-ISSN: 0718-8749
}

№28 diciembre 2011 
Así, la experiencia que abre el acceso al otro, al Mundo y a Dios, se aúna al lenguaje en la forma del dialogo y se hacen ambos el camino de salida del hombre de si-mismo hacia el otro. El lenguaje es para el individuo la experiencia de la revelación y la relación con el otro la expresión de los actos de amor, que a la larga, conducirán los Hombres a la Redención final, que es la experiencia del amor divino, de su perdón y de su eternidad.

Así contribuye Rosenzweig con su obra al rompimiento de los límites entre filosofía y teología, proponiendo una nueva visión del tiempo y de la experiencia e retando a las generaciones actuales a que sigan el camino del diálogo que el mismo articuló y vivió.

Como dice Rosenzweig en las líneas finales de la Estrella de la Redención, “ ¿Hacia dónde se abren las hojas de esta puerta? ¿No sabes? A la vida’’22. Entonces, para ahí caminemos.

\section{BIBLIOGRAFIA}

ROSENZWEIG, Franz. Hegel und der Staat. Druck und Verlag von R. Oldenburg, München und Berlin, 1920 ROSENZWEIG, Franz, ROSENSTOCK-HUESSY, Eugen. Judaism despite Christianity. The "Letters on Christianity and Judaism" between Eugen Rosenstock-Huessy and Franz Rosenzweig. ROSENSTOCK-HUESSY, Eugen (ed.). University of Alabama Press, Alabama, 1969. ROSENZWEIG, Franz. El nuevo pensamiento. Visor, Madrid, 1989. Trad. Isidoro Reguera y Francisco Jarauta ROSENZWEIG, Franz. La Estrella de la Redención. Trad. de Miguel García-Baró. Salamanca: Sígueme, 2006. SCHELLING, F.W.J, Las Edades del Mundo. Cerro Alegre, Santiago, 1993. 